\title{
XIV.
}

\section{Emendationes Arnobianae.}

Quas ante hos quatuor annos emisi Quaestiones Arnobianas criticas, cum spectatissumo harum literarum existumatori prieter nnum locum probatue essent (cf. Liter. Centralblatt 1863, nr. 23), extitit qui, quamris rem ratione et via actam esse concederet, tamen non uno nomine sibi minus probari profiteretur (in „Beiträge zur kritik lateinischer prosaiker" p. 21-31), Theodorum dico Hugium Helvetum. Qui ut libros Arnobianos, si minus continuis, at subsecivis studiis se attigisse fatetur, ita, etiansi hic illic scriptorem egregie emendaverit, usum tamen Arnobianum mirum in modum ubique consentientem et, quanvis multorum lomo sit verborum, tamen artis finibus circumscriptum non satis cognitum et perspectum habet, ut ipsum illud, quo reliquos, quotquot in hoc scriptore emendando versati sunt, notandos censui etian in hunc doctissumum virum cetera acutum cadere videatur. Etenim, si qui scriptor antiquus ex se ipso emendandus est, hic Arnobius ndeo sibi in scribendo constat. ut, nisi ex sanis partibus eam, qua aegris melearis, petiveris medicinam. verear ne futurum sit ut innumeris sanequam locis sanandis acumine et ingenio hand raro abusus esse dicaris. Ut brevis sim, ubicumque acumine ad rectum inveniendum opus erat, bene rem suam egisse videtur homo doctissunus; ubi exacta sermonis cognitione, non videtur. Velut p. 29 me increpat, qui VI, 14: (deum) cum scius et cerlus sis tui esse operis et digitorum parlem spreta Ursini correctione quae est partum, ,acutius quam felicius" maluerim partem i. e. per artem; quippe locos Lactantianos a me ipso allatos, quam recte Ursinus correxisset, me docere 
debuisse. Legitur autem apud Lactantium (Instit. IV, 1. 2) digitorum figmenta et (II, 2. 12): homo quasi parens putandus est, per cuius manus nata sunt (simulacra). Praterea Hugius auxilio vocat Petronium (118): negue concipere aut edere partum mens potest. At quid illa digitorum figmenta ad rem? Fingere digitos scio, parere nescio, hominem parentem dici scio i. e. patrem, matres homines dici nescio, meutem (i. e. rem femininam) partum concipere et edere scio, digitos matres ignoro. Quod VII, 18 pro mints scribendum censui muneris, ineptum Hugio visum est; quem enim unquam de sanguine muneris deis oblati audivisse? At si quod deis offertur munus sanguine suo deos placare dicitur, ipsum illud munus vitam et singuinem hatbere $i$. e. victimam esse intellegitur. Atque recte me munus quidem substituisse M. Hauptius (in Herme II, 11) consentit, traiectum minus, id quod Hugio placuit, sententiam alsurdam efficere monens. Denique quace de $I I, 55$ disputavi, quam essent incerta, cum ne de codicis quidem lectione constiret, bene gnarus, ut censori meo Lipsiensi recte non satisfecerunt, ita iam mihi ipsi cum Hugio minus probantur; quamquam quae Hugius suo Marte de hoc loco statuit, tatm sunt infirma, ut ne ipsum quidem Hugium in eis adquiescere quovis pignore adfirmaverim; cum enim prater hunc locum illud quinimmo potins nisi IV, 18, ut $p$. 8 et 9 exposui, in lihris Arnobianis non legatur, perdoctum melercules librarium fingit Hugius, qui ex hoc loco glossam illam adscripserit! Quae autem addit de verbis per voluntutem ut simul intellegatur et malis eum causas dare et miseriarum esse innumerabilium conditorem, ne intellego quidem. Quid enim? Verba per voluntatem coniungenda putavi cum intellegatur, id quod factum esse ille se mirari fatetur. Esto: coniungamus cum superioribus: sine deo nithil fieri per voluntatem. At per cuius voluntatem? si quid per voluntatem fit sine deo, humanam voluntatem esse apparet, non divinam; quid enim est: sine deo aliquid fieri per eiusdem dei voluntatem? Ergo humana voluntas est; igitur timen de voluntate divina. Quae cum ita sint, recte per voluntatem asl simul intellegatur coniunxisse videor. Restat, ut Hugius me falso contendisse doceat, eius modi dictionem Arnobianam non esse.

Haec sunt quae quasi pro domo praefanda halbui; sunt autem omnia, in quibus Hugium se mecum non consentire professus est. Neque profecto, utpote qui mea admirari non soleam, tanti duxis- 
sem, ea tueri, quae multis nominibus manca sunt et parum laudis habent, nisi Hugius apertissuma me non vidisse, me miracula vendere (,höchst kurios ist sein vorschlag“) nescio qua dicendi insolentia adfirmasset. In tripode, puto, unus sedet Apollo.

Arnob. I, 3 extr.: immunis enim antiquitas malis ab his fuit, cum etiam fumina cognoverimus ingentia $l i m i n$ is inhorruisse siccatis? Omnes quod sciam viri docti aut limis substituerunt aut limfis. Quorum alterum et a codicum lectione longius abest et recto iudicio caret. Limis autem fuerunt qui recte dici negarent, ut ipse lamis substituendum esse olim opinarer (cfr. Sulpicius Severus Cbron. I, 33 ed. Halm.). Sed recte emendatum esse limis Statius (Tbeb. IV, 698) probat:

viridisque comis exhorrnit humor.

protinus Inachios haurit sitis ignea campos;

diffugere undae, squalent fontesque lacusque

et cava ferventi durescunt fumina limo.

De verbi inhorrescere notione cfr. eundem Statium (Theb. XII, 167): at illis

horruerant lacrimae.

I, 6 in.: quamquam ista quae dicitis bella religionis nostrae ob invidiam commoveri non sit difficile comprobare post auditum Cleristum in mundo non tantum non aucta, verum etiam maiore de parte futuriarum compressionibus imminuta. Pro corrupta illa voce editores ad unum omnes correxerunt furiarum. Debebant, opinor: maiore de parte $s$ u furiarum compressionibus imminuta. Quam emendationem cum ipsa codicis lectio suadet, tum usus Arnobianus poscere videtur. Sic I, 1: sui vitio; I, 53 med.: in exigua sui parte; II, 27 med.: sine sui mulabilitate; II, 38 extr.: integritatem sui; VII, 3, in.: debet habere causam sui; VII, 16 in.: sui natura; VII, 29 in.: non habebit expositam sui causam; VII , 44 extr.: partem sui extremam. Uude male fecerunt, qui spreta codicis lectione ingenua II, 42 extr.: virilitatem sui populo publicarent aut utilitatem substituerunt aut vilitatem; Plura de loc usu leguntur apud. Lud. de Jan Prolegom, ad Macrobium p. xxxix.

I, 8 extr.: nec vir prudens (Plato) extimuit humani generis subversionem, cladem, ruinas, interitus funera rerum innovationem vocare et in iuventutem his quandam redindegratis viribus comparari. Editores aut in eicere aut cum Ursino etenim scribere ma-

Pbilologus. XXYI. Bd. 4. 
luernnt; unus dubitavit Hildebrandus. Recte. Scribendum euim et inde, quemadmodum etiam in codicibus Curtianis $(V, 17,8$ ed. Zumpt.) in pro inde exaratum est. Eadem medicina utendum erit II, 48 in.: inde consimiliter hic quoque, cum animas renuamus dei esse principis prolem, non continuo sequitur ut explicare debeamus, quonam parente sint editae. Cod. in, quod reliqui editores eiecerunt, Hildebrandus plaudente 0eblero in sic mutare maluit.

I, 14 extr.: quemadmodum enim res agi et usque ad hoc tempus genus quis duraret mortalium, si non omnia, quae usus ut posceret, subministraret fertilitas rerum? Ita cod. Recte Ursinus: quiret durare, quod huius aetatis editores contempserunt omnes. Quae usus sibi posceret cum Hildebrando Oeblerus; Hugius p. 25 ut eicit, ut dittograpbia ortum. Equidem puto quae usus vitas posceret scribendum esse. Simillumus locus Lucreti, quem Arnobius solet imitari (VI, 10: ad victum quae flagitat usts). Iutila autem verba in codice non pauca inveniri postera docebunt.

I, 15 in. Falso bucusque interpunxerunt locum, sic distinguendum: sed fuerunt aliquando nonnulla in necessitatibus tempora: et relaxata sunt abundantiis rursus. Contra cet.

I, 17 extr. incredibilia quaedam cum Hildebrando conatur 0eblerus. Redeundum ad ea, quae recte servavit 0rellius, ut tamen iactet scribas pro iaciat. Hildebrandum ne de verbi iactari forma nescio qua deponentiali hariolaretur deterrere debebant I. Haupti Quaestiones Catullianae p. 56.

$I, 23$ med. Hugius, ut veterum editionum lectionem $a b$ su $\mathrm{mmi}$ culminis decidisse fastigio tueretur, non ad Vergilium debebat provocare, sed ad ipsum Arnobium II, 24 med.: ex altissimi culminis se rursum praecipitasse fastigio. Plura circumspicienti aderit Nuetzell ad Curtium IV, 8, 8, p. 195.

I, 29 med. In codice est: nonne huic (deo) omnes debemus hoc ipsum primum, quod sumus, quod esse homines dicimur, quod ab eo vel miss a e vel lapsae caecitate huius in corporis continemur? Quam scripturam editores ad unum omnes mutarunt in missi vel lapsi; missae enim et lapsae quid sibi velint se non videre adfirmat Hildebrandus. Atqui, modo ipsum scriptorem consulas, res planissuma est. Ut singulis exemplis defungar, legitur II, 13 extr. quid sumus homines nisi animae corporibus clausae? Has autem animas, quibus homines simus, ad corpora $l a b i$ statuit II, 16 in., 
videlicet ex caelo, ut legitur II, 18 in.: neque cum animis artes caeli ex penetralibus ceciderunt, vel II, 39, in.: rex mundi.... ex se genitas huc animas misit. Inde apparet de animis humanis, i. e. de ea nostri parte dici, qua homines sumus. Atque re vera tale quid subodoratus est Oellerus, animae nostrae excidisse et pro continemur scribendum esse continentur suspicatus. Sed cum recto iudicio incedere nesciret et genus scribendi Arnobianum plane ignoraret, melius sane fecit quod ad lapsi et missi redire maluit. Solet autem Arnobius adpositiones, quas dicunt, ita enuntiationi, cui alias adiungi solent, iniungere, ut praedicatum a primaria notione pendeat, de qua re satis erit relegasse ad ea quae Hildebrandus $\mathbf{I}$, 6, p. 15 congessit. Unde emendandum est: quod ab eo vel missae [animae] vel lapsae caecitate huius in corporis continemur. Erat enim in codice archetypo, quem multis notis usum fuisse in quaestionibus Arnobianis docui, missae ae vel lapsae, unde quomodo ea profecta sint quae in cod. Parisino leguntur, manifestum est.

Eadem via succurrendum erit 1, 31 med., cui loco vix dici potest quam perversis artificiis adbuc mederi conati sint. Legitur enim in codice: prima enim tu causa es, locus rerum ac spatium, fundamentum cunctorum quaecunque sunt, infinitus, ingenitus, immortalis, perpetuus, solus, quem nulla deliniat forma corporalis, nulla determinet circumscriptio, qualitatis expers quantitatis, sine situ, motu et habitu. Quo loco viri docti recte haeserunt in vocibus qualitatis expers quantitatis cui dissolutioni ant traiectis verbis aut male dispunctis subvenire voluerunt. Equidem facilluma medela ita rem expedio: qualitatis expers, [expers] quantitatis, ut hoc etiam loco librarius alterum vocabulum contiguum omiserit.

Nescio an simili quodam modo subveniri possit loco qui est I, 34 extr. Ibi cum cod. haec praebeat: at vero Iuppiter, ut vos fertis, et patrem habet et matrem, avos avias, fratres, nunc nuper in utero matris suae formatus, absolutus mensibus et consummatus decem ignotam sibi in lucem sensu ir ruisse vitali, ab Ursiuo inde correxerunt irruit se. Malim: avias, fratres; nunc nuper in utero matris suae formatus, absolutus mensibus et consummatus [dicitur] decem ignotam sibi in lucem sensu irruisse vitali. Erat enim in codice arclietypo: et consummatus $\overline{d r}$ decem.

De loco $I, 45$ extr. optume meruit Hugius p. 28. Restituendus enim laud dubie loci ordo et conformatio; quam enim in bis 
etiam rebus sibi constare soleat Arnobius, II, 8 med. te docebit. Quamquan codicis vestigia diligenter premenda censeo, ut spreto illo quod Hugius proposuit et iam [ambulare atque exilire] coeperant malim et iam [ambulare nullius illis] operis res erat, ubi quae uncis inclusi Arnobiana esse non magis adfirmo quam quae Hugius addidit. Hoc tamen tenendum erit, nullius operis res est ab usu Arnobiano non ablorrere. Quae Hildebrandus quidem ad I, $10 \mathrm{p}$. 20 congessit, nihil faciunt ad rem; legitur autem I, 25: communis officii res est; et II, 57 extr.: res est supervacui operis.

Aegre accedo ad I, 51, in.: Curionem aut pontificem maximum, quinimmo dialem, quo deius est, flaminem isto iure donavit? Corruptan esse codicis scripturam patet, quamvis quae ea sit scriptura, Valleno, ut eruat, relinquendum erit. Quod scripsi, se legisse testatur Hildebrandus; grod eius est Crusiana collatio praestat. Nihil utrimque sani. Hoc tamen certum est, nihil in illis verbis dubiis inesse posse nisi Dialem flaminem proprium lovis esse. Quod munus per quinque et septuaginta annos cessasse Tacitus auctor est (Ann. III, 58 ibiq. Nipperdey). Qua auctoritate usus Arnobium scripsisse putaverim: quo diu usus est.

I, 55 extr.: immo quia (gentes) haec omnias et ab ipso cemebant geri et ab eius praeconibus, qui per orbem totum missi beneficia patris et munera dis animis hominibusque portabant, veritatis ipsius vi victae et dederunt se deo nec in magnis posuere dispendis membra vobis proicere et viscera sua lanianda praebere. Praeter illud dis etiam hominibusque corruptum esse ipsa res docet. Cui enim praeter homines portaverint? Itaque huic quidem vocabulo que adiungi non poterat. Emendo, non curans quod secunda in codice manus adscripsit numerandis vel munerandis: beneficia patris et munera divinis animis ominibusqa e portabant. In codice archetypo erat $d \bar{z} s$; ominibus autem et hominibus etiam $I, 35$ in codice confusa sunt.

I, 61 med.: si oporteret ita fieri quemadmodum dicitis, ita fortasse fecisset; quia non oportuit, aliter fecit. Qua re isto voluit et illo genere noluit, latentaliter involutae et vix ullis compraehensibiles causae. Cum omnes a Meursio inde editores latent taliter scripsissent, primus Hoftmannus (Archiv für philologie und pädagogik xıII, p. 153) posterius vocabulum ut dittographia ortum eiecit, quem inscius secutus est Hugius (p. 25), iam rem expedi- 
tam pollicens. At facili, nisi fallor, opera manum Arnobianam restitues, modo scripseris latent fataliter involutae.

II, 1 extr. unum vocabulum excidisse videtur, quo restituto Arnobius Arnobio redditur: numquid adrogantiae supercilio tumidus iniurias et contumelias passim sine [ullis] ullius personae discriminibus inrogavit? Cfr. III, 1: neque apex ullus ullius praetermissus est quaestionis.

II, 5 med. Hild. (II, 4 0rell. et 0ebl.): quid enim? quae vos negatis vera esse, apud vos liquent (cod. liquet), oum imminentia et nondum passa millis possint rationibus refutari? Interpretes in boc loco inlustrando nihil agunt. Excidit aut accusativus aut infinitivus aut utrumque.

II, 7 in. Oehl. (II, 6 extr. Orell. II, 2 extr. Hild.) postquam antiquiores editores rectam, qua suum Arnobio reddatur, viam monstraverunt, mirum in modum nuperi editores tergiversati sunt. Atque primum quidem Meursius melius interpunxit, vocabulum primum cum antecedente sententia coniungens. Ita enim et ratio et usus Arnobianus poscit. Primum enim deum Cbristianorum innumeris locis dicit, velut II, 29 extr., II, 72 med., III , 2 med. Scribendum antem: nunquamne illud vulgatum perstrinxit aures vestras, sapientiam hominis stultitiam esse apud deum primum? Eoqui ipsi penitus perspicitis vos ipsos? si quando de rebus disceptatis . ... tutari? De voce ecqui cf., si tanti est, II, 29 extr. II, 30 med., de sententia Minuc. Fel. V, 5 Halm.: satis satisque prudentes iure videamur, si secundum illud vetus sapientis oraculum nosmet ipsos fumiliarius noverimus.

II, 9 med. Recte Hildebrandus in verbis qui cunctarum originem ignem esse dicit aut aquam, non Thaleti aut Heraclito credit? omissum esse rerum statuit; scribi tamen debebat rerum cunctarum, non cunctarum rerum. Cfr. I, 25 med. II, 74 extr.: ipse rerum cunctarum pater. Etiam III, 2. med. malin: pater rerum ac dominus constitutor moderatorque cunctarum.

Inveteratum ulcus baeret II, $11 \mathrm{in}$. idque tam manifestum, ut bucusque latuisse vix credibile videatur. Arnobius enim gentes increpat, quod, cum ipsae placitis et opinionibus philosopborum fidem babeant, Christianos in Cbristo adquiescentes vituperent. Iamque etbnicos Cbristianis ita opponit, ut in singularum enunciationum partibus prioribus illos id sibi ipsis concedere dicat, quod Cbristianis 
licere negent. Iniquitas, pergit, haec quanta est, ut . . vobis velitis dari, quod ita ab illis (philosophis) dicatur, accipere, vos ea quae proferuntur a Christo, audire et spectare velitis. Vides, singula singulis opponi; vos autem illud nec verbum labet cui opponatur nec rem, ut sive a sententia sive ab arte grammatica plane absonum sit. Quid multa? Scribendum est: nos ea quae proferuntur a Christo audire et spectare nolitis.

Ursinus cum II, 13 extr. recte correxisset abstineatis (cod, abstinetis; ita tamen cum reliquis editoribus locum interpunxit, ut perversa exiret sententia. Scribe: vos enim non omnes pro illarum (animarum) geritis incolumitatibus curas? non quod vitiis omnibus et cupiditatibus abstineatis: metus ille vos habet, ne velut trabalibus clavis adfixi corporibus haereatis. Atque in extremis verbis ea spectat, quae philosophi quidam antiqui de animorum flagitiorum labe inquinatorum sorte post mortem futura statuerant. Cf. Plat. Phaed. p. 81 et 82 ibiq. Wyttenbacb.; Moser ad Cicer. Tuscul. I, 30, 72, praecipue autem Augustin. de civit. dei XII, 26. Ut nunc quidem locus interpungitur, non omnes i. e. plurimi animarum curam habere dicerentur, id quod contra ipsum scriptoris consilium est.

II, 15 in.: a quibusdam dicitur viris ... animas immortales esse, deo rerum ac principi gradu proximas dignitates cum cod. babeat, nescio cur omnes, quod sciam, editores dignitatis maluerint, „familiare" codicis vitium se sustulisse opinati. At dignitates sensu concreto dici pro ordine dignissumo docent Fabri et Heerwagen. ad Liv. XXII, 40, -4, Halm. ad Cicer. pro Sestio 51, 109. Ipse Arnobius I, 28: potestates; II, 38: tyrannos, dominos, alias potestates, I, 46: Christus interiorum potentiarum dens; I, 64: nobilitatibus.

$I I, 15$ extr. codicis lectio restituenda erit: nec, quot in orbe sunt homines, non sunt sententiae totidem. Nimirum qui negationem eiecerunt aut miris quibusdam coniecturis cedere iusserunt, scriptorem acerbissuma simulatione usum esse obliti videntur. Illud enim non coniungendum est cum vocabulo totidem, ut sententiarum numerum non imparem simulet numero bominum.

II, 16 med.: Vultis homines in situm typhum superciliumque deponere, cum in codice legatur institutum, quam vocem misere et frustri se torserunt qui tueri conati sunt, ut emendetur, primum II, 7, 0ebl. (II, 3 Hild.): nos ita caecos et superbos nescio quae 
res protulit, tum id suadere videtur, quod spiritus (isti spiritum Hildebrandus coniecit) superbiae notionem apud Arnobium quidem non habet, typhis autem apud eum frequens est (cf. II, 12 extr.; II, 19 in. II, 29 med.). In subsequentibus pro furore 0elleriano fervore usus Arnobianus poscit. Cf. Hildebrand ad I, 18.

II, 25 in.: haecine est anima docta illa, quam dicitis, immortalis, perfecta, divina, post deum principem rerum et post mentes geminas locum obtinens puartum et affuens ex crateribus vivis? Prima quae scripsi verba editioni Basileensi debentur; in codice est haec inteslamina. Sed ne sic quidem scriptoris meutem intellego, quam vereor ut interpretes quotquot sunt explicuerint. Geminae enim

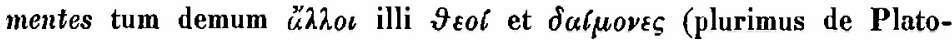
nica illa - cf. Timaeum Platonicum p. 81 C. D. - quam Arnobius spectat sententia est Astius ad Platon. Plıedr. Commentar. Tom. X,

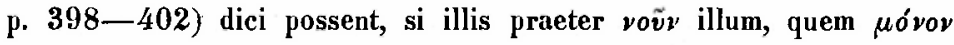

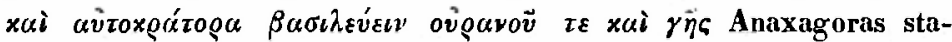
tuit, ullus esset providendi locus relictus. Illos tamen deorum ordines hoc loco intellegi praeter dubium est. Mihi quiden, nisi ad conjectandum confugias, res expediri non posse videtur: itaque auctor sum ut scribatur: post daemones et genios. Daemones quamvis quos dicat nullo loco Arnobius planis verbis profiteatur, plurimi tamen scriptores ecclesiastici omnes etbnicorum deos dicere solent; $\gamma \varepsilon \nu^{\prime} \eta \eta_{-}$

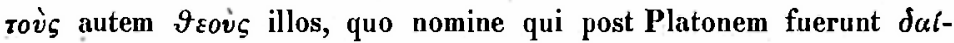

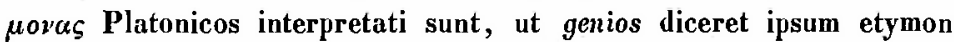
suadere poterat.

Retracto sententiam olim in Quaest. Arnob. critic. p. 4 de $I, 28$ in. latam, qua ne, quod antea nae scribere solebant, adfirmativum Arnobio obtrudendum pravo iudicio usus statui. Jam enim aliquid intercidisse censeo. Cum enim in cod. legatur: ne videamus, lanc quidem rocem non suo loco relictam, alteram eamque simillumam a librario praetermissam putaverim, ut ita ipse Arnobius scripserit: ac ne tamen instructi non plenius abeamus, videamus ne andiamus a vobis ect.

Eodem capite medio duobus locis Oehlerus male codicem secutus est. Nam una enim causa res duas efficere ac sibi contraria non potest absurdum est; immo potest duas vel plures res efficere, at non eas sibi contrarias; ita enim corrigendum esse reliqui editores perspexerant. Plane tamen alia res est in sequentibus: quodsi 
animae quas vocatis membrorum impediuntur obstaculo, quominus artes suas atque antiquas reminiscantur. Acceperant enim artes illas antequam in hanc terran mittereutur, ut quomodo animae suas at $q$ ue antiquas reminisci dici possint non intellegatur. Itaque aut antiqua scribendum est aut, id quod malo, substantivum neutrum plurale intercidisse videtur. Quamquam II, 26 med. ipse Arnobius: oblivionem priorum nullam pati.

II, 29. med.: Recto iudicio egit Elmenhorstius, quod egregin Meursii coniectura probata locum sic constituit: cum enim vos oporteret, viros recti atque integritatis auctores, typhum et adrogantiam frangere, quorum alis (cod. quorum malis) extollimur et inanium distendimur vanitate. Etenim admissa voce malis scribendum fuerat quibus malis. At in sequentibus haeret ulcus: non tantum, pergitur, accidere mala ista censetis, verum, quod gravius multo est, addidistis causas, quibus et vitia crescerent et inemendabilis nequitia permaneret. Apparet enim duobus nominibus magistratus etbnicos vituperari, primum quod typbun et adrogantiam grassari patiantur, tum quod vel materiam vastioris incendii subministrent. Plane tamen contrarium in verbis, ut nunc quidem feruntur, Arnobianis inest : non tantum coerceri illam norum labem, sed etiam ali. Quae cum ita sint, alteram particulam negativam intercidisse patet: non tantum accidere mala ista [non] censetis, verum e. q. s. Idem accidit II, 49 fin., ubi restituendum: et [non] modo non parvi (cod. et modo non parvi, editt. praeter Oeblerum nec modo non parvi), sed esse mullius existimandum est mominis. De omissa particula negativa conferri poterunt $Z$ umpt. ad Curt. IV, 40. 17, C. W. F. Müller. in Fleckeiseni Annal. 89, p. 279.

II, 33 in. Hildebrandus codicis lectionem commodi facimus sensu, quo amamus nos omnes tueri debebat alio eiusdem scriptoris loco, qui est Il, 37 extr.

II, 35 med.: est enim quod obstare eius voluntatibus possit aut quod esse voluerit, non necessario sequatur ut fiat? An numquid nosiris ex divisionibus colligemus, quid aut fieri possit aut non possit? Duplex vitium latere videtur. Prior enim sententia ne intellegitur quidem; restitue: $u t$ quod esse voluerit non necessario sequatur ut fiat? In reliquis malim divinationibus; quamquam nescio an nostris ex divisionibus interpretari possis: cum in tam diversa abeant nostra de his rebus iudicia. 
II, 35 extr.: si sunt prolati et geniti, et ordinis sunt posterioris et temporis ut emendetur, quamvis cum ipso codice Hildebrandus et Oeblerus et Hugius reclament, subsequens sententia flagitat, utpote eoden modo conformata. Nihili sunt quae Hildebrandus de omittenda particula congessit ad II, 25, p. 162. Neque vero recte de bac re iudicat Hugius p. 27, ubi III, 28 extr. aliquid intercidisse (id quod et ipse aduotaveram) recte suspicatus: aut faciunt restituit. Quippe aut si faciunt debebat. Plane enim in bac re Arnobius cum optumis scriptoribus consentit, ut Hugium ab illo quod tulit iudicio avocare debuerint quae Madvigius in Opusc. acad. I, 54 egregie exposuit. Sero Hildebrandus ad meliora rediit, qui cum in adnotatione illa perverse de loco $V, 8$ iudicasset postea cum quo si stat in textu exhibere maluit.

II, 36 in. Arnobius postquam deos immortales non natura, sed dei voluntate ac munere esse docuit, idem de animis lumanis comprobaturus pergit: quo igitur pacto immortalitatis largiter est donum dei certa prolatis et animas hoc pacto dignabitur immortalitate donare. Ita codex. Recte editio princeps largitus; in reliquis nilil sani. Emendo: quo igitur pacto immortalitatis largitus est donum diis die certa prolatis, et animas hoc pacto dignabitur immortalitate donare. In capitis enim antecedentis fine id quod introitum et vitae incipientis exordium habuisset, idem necessario etiam exitum habere posuerat; iam in bac quidem re animas humanas nilil a deis istis differre pergit. Ut enim certa quadam die anima, ut cum ipso Arnobio loquar, in hominem nascatur, ita etiam deos certa quadam die natos esse. Quod quid sit, ipse Arnobius docet VII, 32, ubi de natali Telluris die verba facit et deos ex uteris prodire adfirmat. De ea re quid antiqui homines statuerint docebunt te Marquardti Antiquitt. Roman. IV, 148; Prelleri Mytholog. Rom. p. 139 edit. I.

I, 36 extr. cum Arnobius ad Christi ipsius testimonium provocet, editores ad gnosticam Carpocratis aliorumque doctrinam, ut, quae in extremo hoc capite leguntur, explicarent, confugere maluerunt. Nos scriptoris verba prementes primum $S$. Iohaunem testamur, apud quem (Evangel. VIII, 44) haec leguntur: $v \mu \varepsilon i \tilde{s} \dot{\varepsilon x}$ tõ

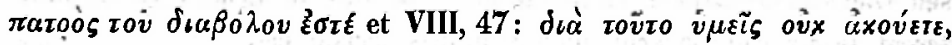
öt Ex rou $\vartheta_{\varepsilon} \sigma \tilde{v}$ ovx $\dot{\varepsilon} \sigma \tau$. Haec respiciens Arnobius: discite, in- 
quit, ab eo qui novit et protulit in medium Christo, non esse animas regis maximi filias. Quae auten subsequuntur: nec $a b$ eo, quemadmodum dicitur, generatus coepisse se nosse atque in sui nominis esse sententia praedicari, in bibliis sacris non leguntur, sed ipsius Arnobii sunt suum iudicium alienae auctoritati suo more ant interponentis aut subiungentis. Sed ea verba ut recte intelligantur, is tenendus est scriptoris usus, quo, etiamsi multorum fere verborum esse soleat, sententian aliquam, quam accuratius exponere poterat, paucis nonnunquam verbis quasi praetereundo inserit, cuiusmodi ea sunt verba (die certa) quae in huius capitis initio restituimus. Id quod etiam hoc loco factum est. Si enim, ait, ab ab ipso deo profectae essent animae, ex eo inde tempore (dicit enim coepisse) originis suae meminissent atque divinitatis sublimitate gloriarentur necesse erat. Jam vero animas lumanas cum deis, quos ipse quidem esse negat, sed, quo melius vulgarem gentium opinionem refutare possit, esse dat et largitur, hoc loco ita de immortalitate comparat, nt ntrosque eadem sorte esse doceat. Atqui de deis I, 28 med. in re plane simili laec leguntur: per quem (i. e. deum patrem) esse et habere substantiam sui numinis maiestatisque coeperunt; neque multo post: cuncta in sui nominis naturam prosiluisse ait. Quae cum ita sint, emendo: nec ab eo, quemadmodum dicitur, generatas coepisse se nosse atque in sui nominis esse substantia praedicari, quae verba ita construenda sunt: nec coepisse se ab eo, quemadmodum dicitur, generatas nosse atque in sui nominis (i. e. animarum a deo generatarum) substantia esse praedicari (coepisse). Sententiae et substantiae notae non multum difterunt: $\overline{\text { snia }}$ et suba. Quod autem praedicari cum nominativo cum infinitivo coniungitur, plane Arnobianum esse docebit Hildebrand. ad VII, 46.

I, 41 med.: "t alias a liar $u$ m raperent, ut canibus mos est et vulturiis, portiones scribendum esse res ipsa docet; cod. alia. In sequentibus excidit aliquid, patrimonia puto (cf. II, 50 med.); [patrimonia] subigerent dentibus et crudelissimo ventri darent. Neque dubitandum quin recte Ursiuus emendaverit: et in tam saevis et horridis moribus sortem suam flerent.

Sub eiusdem capitis finem non linis Heraldus suspicari debebat sed lineis. Cf. Huebner. in Herme I, 350. In ipsis verbis: innecterent lineis colla acerbissuma vanitatis irrisio est.

II, 44 in. lege: ut eas prohiberet, absconderet. Eodem capite 
medio excidit malorum: aniversorum [malor $u m$ ] non alius quam ipse est causa, cf. II, 54 seqq. Idem accidit II, 46 med., ubi corrigendum: quod minus [ab]esset a recto aut quod minus esset [summotum] a recto: cf. VII, 35 extr., et sub finem capitis: qui nulla alia de cansa esse [se] intelligit procreatum. Eodem loco cum cod. Parisino retinendum erit quamvis latens.

$I I, 47$ med. pro mihi istud necessario sequitur maluerunt nihil; equidem minime.

$I I, 53$ med, tantum abest ut cum Hugio p. 24 verba ineptissime fatuae (ita codex) eicienda putem, ut facili opera ita loci integritatem restitui ceuseam: Sed stulte istud credimus. Ouid ad vos, [si] ineptissime, fatue? Quippe contiguae utriuque literae particulam conditionalem absorpseruut.

II, 56 med.: mundum quidam ex sapientibus existimant nequo esse siccatum neque ullo esse in tempore periturum. Ex qua codicis scriptura non natum, sed sic creatum eruendum videtur.

II, 59 in. non dubito quin scribendum sit: si praesto est quod libuerit scire et in aperto verum et in scientia constitutum. Hucusque fertur in aperto rerum, quod latinum non est. Cf, si tanti est II, 51. extr.; II, 72 extr.; Hildebrand. ad V, 29.

II, 59 in. Codex: Dicite quid sit quod grandinem torqueat, quod guttatim faciat pluviam labi, quod imbresue nives plumeas et folgora (a m. sec. foliora) dilatarit, in quibus verbis quid folgora vel monstrum illud vocabuli foliora sibi velint, frustra docti homines laboraverunt ut divinarent. Fuerunt, qui nullo consilio Aeoli ora, vel foliola coniectarent, fuerunt qui, velut Heinsius, ad

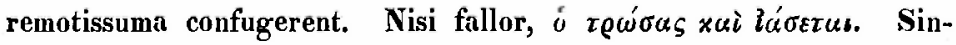
gula enim vides vividissumis coloribus depicta, ut quae singularum rerum propria sunt, felici quodam narrandi artilicio exhibeantur: grandinum tormenta, pluviarum guttae, imbrium plumae. Sequitur ut aut substantivum cum adjectivo deesse statuas aut adiectivum cum nivibus iungendum. Atqui fulgura hoc quidem loco ineptissuma sunt. Ergo adiectivum aliquod desiderari apparet. Scribe: quod imbres in nives plumeas et vellereas dilatarit. De nivium velleribus cf. interpr. ad Vergili Georg. 1, 397; Wernsdorf ad Severi carm. bucol. 87 (P. L. M. II, p. 225): quam densis fluitant velleribus nives. 
II, 59 paullo post: quae est causa, quae ratio, ut maria sola sint aut terrarum hae dulces aliae sint amarae vel frigidae? Ipsa opposita aliquid in codice deesse docent. Scribe: ut maria sola [salsa] sint aut terrarum [aquae] hae dulces [vel calidae], aliae sint amarae vel frigidae.

Neque minor in eodem capile medio lacuna est. Codex: (quid in mundo faciunt) avenae, quid lolium, quid herbarum aut fruticum aut adulentia (a. m. emeudatrice adolentia) naribus aut tristia in odoribus semina? Quod quomodo nuperrumi editores ferre potuerint, non minus mirum est quam quod vetustiores aut suaveolentia aut redolentia e coniectura admiserunt. Interrogatur enim, quid nocuae et molestae res in mundo faciant, ut sive innocuis sive suavibus locus non sit. Praeteren ipsa en quae in codice relicta sunt, aliquid desiderari clamant. Restitue: aut a[d tactum] dolentia, naribus aut tristia in odoribus semina.

Eodem capite extr. in codice haec leguntur: ipsa deinde haec quid sint, sapor dico id est sapor et cetera qualitatum distantias quibus ex rationibus ducant. Ex ipsa horum verborum mira sane specie librarium scribendi laborem post prius illud sapor paullulum intermisisse patet, ut tamen, si minus verba, at sententiam loci exarandi memoria teneret. Iam cum opus intermissum denuo reciperet, aliquid sequi, quo priora, quae essent, explicarentur meminerat; itaque non inspecto exemplari pergebat dico (etenim se saporem modo scripsisse opinabatur); tum cum ad exemplar redisset, id est sapor, non deletis vocabulis. antecedentibus, ex eo subiuuxit. Itaque expunctis illis vocabulis scriptori suum ita restituetur: ipsa deinde haec quid sint, sapor et cetera e. q. s. Quamquam 11, 60 extr. res vestra in ancipiti est, salus dico animarum vestrarum legi bene scio. At praeterquam quod in cod. dici legitur, unde dico coniecerunt, diversa utriusque loci conditio est. In hoc enim res gravissuna et quae in superioribus non commemorata sit, extollitur, in illo res levissumae et quae paullo ante nominatim appellentur, explicandi caussa subiunguntur, ut, qui est Latinorum hominum usus, accusativo (saporem) opus fuisset, in altero (si quidem, de qua re mihi quidem non liquet, recte emendaverunt), recte scribatur salus; quippe dico exbortantis esset et graviter monentis.

II, 60 in. Codex: cum igitur... neque dicere neque explanare possitis, quid sit factum aut quare aut cur oportuerit non esse. 
Hiulca verba sic restituenda esse: aut cur oportuerit [esse aut] non esse docet locus geminus II, 61. med.: remittite haec deo atque ipsum scire concedite, quid, quare aut unde sit, debuerit esse a $t$ non esse. A subsequentibus abstinendum erit; diversa enim de codicis lectione referuntur.

II, 61 in. Scribe: quid prodest, inquit, vobis investigare, conquirere, quisnam hominem fecerit. Cod. et editt. omnes: quid est.

II, 61 med. Cod.: vestris non est rationibus liberum, implicare vos talibus et tam remota in utilitate curare. Recte priores editores in hoc loco haeserunt; qui cum inutilitate ferri non posse perspicientes inutiliter scribendum censuissent, Hildebrandus exemplis a re plane alienis usus codicis lectionem revocavit plaudente Oelılero. Equidem suaserim: et tam remola involuta antiquitate, quemadmodum solet Arnobius.

$I I, 63$ in. cum in codice legatur: potestis enim scire, quid sit cum eius nimis actum plurimi editores cum eis animis maluerunt, Rigaltius cum cunctis animis. E codicis autem reliquiis eruendum videtur: cum einsmī ăs, i. e. cum eiusmodi animis.

II, 67 in. nobis intenditis aversionem et religionem priorum. Ita codex; ex religione ed. princ., a religione Ursiuus, aut hoc aut illud reliqui. Aversio autem ex religione ut latinum non est, ita codicis vestigia nimis reliquerunt, qui a religione emendaverunt. Quae in sequentibus capitibus leguntur, ubi Romanos a maiorum moribus et religione defecisse docet, faciunt ut ita emendatum velim : aversionem [a more] et religione priorum.

II, 67 med. Meursium et Lipsium egregie restituisse: in penetralibus et colinis comprobant ea quae leguntur apud Corssenium (Ausspr. I, 255) et Marquardtium (Antiquit. Rom. IV, 209).

II, 69 extr.: quisquam hominum scicbat .... in fulminum casibus aut extorum al iquid significaretur in venis? optume Hoffmannus (1. l. p. 157) restituit: extorum mali quid.

II, 70 med. Codex: rursus vero si Liber, Venus, Diana, Mercurius, Apollo, Hercules, Musae, Tyndaridac, Castores, ignipotensque Vulcanus Iove patre sunt proditi et genitore Saturnio procreati, antequam memoria, quam Alchmena, Maia, Iuno, Latona, Leda, Dione cum et Semela Diespetris factae sunt compressionibus fotiae, nuspram et hii gentium nec in aliqua parte rerum $f$ uis se naturae, sed ex conventu Iovis inseminati et nati sunt et aliquem sensum 
sui habere cooperunt. Exbibui verba qualia Hildebrandus se in codice invenisse scripsit; Oeblerus suos auctores Luneburgenses secutus cum et semel a Diespetri in Parisino esse adfirmat. Haeserunt viri docti, ut par erat, in infinitivo fuisse, qui unde penderet frustra circumspiciebant. Itaqne praeter Oeblerum, qui fuissent nullo sensu substituit, uno omnes consensu fuere, et postea, ne Oeblero quidem excepto, tum et Semela scripserunt. Illud quomodo, nisi praeter codicis auctoritatem, in quem quomodo fuisse pro fuere inrepere potuerit non intellegitur, fieri potuerit nescio; hoc cur fecerint apparet. Quippe Semelam tempore extremam ab Arnobio dici perspexisse videutur. At id ipsum addita particula et innui praeterviderunt, ut particula tum non modo possis carere, sed tum atque et iuncta alterum alteri officiant. Quae cum ita sint, pro cum scribendum esse quam mihi quidem certissumum est. Alteri loco, nisi ipsum illud, ex quo infinitivus fuisse aptus fuerit, verbum intercidisse statuas, vix erit quomodo succurras. Verbum tamen ipsum quod fuerit non praestiterim; periclitor: nusquam et ii [intelliguntur] gentium nec in aliqua parte rerum fuisse naturae. Dubitabundus Alcumenam propono: quid dubitem, sciunt quicumque veteres codices noverunt.

II, 71. extr. genitor Latinus cuius? Albani oppidi conditoris, cum aperte falsum esse diu intellexissent, rasurae, quae est in codice post voc. genitor, quid subfuerit egregie Hildebrandus odoratus est. Quamquam non genitor gener Latini is cuius, id quod Arnobianum non est, sed genitor gener Latini cuius? debebat. Nam Latimus ex praecedentibus fluxit.

II, 76 extr. quinimmo edocti sumus minas omnes quaccunque sunt parvi ducere atque aestimare fortunae, ac si quando ingruerit vis quaepiam gravior, quam finem necesse sit consequi, adscribere infortunio voluptatem, nec timere nec fugere, quo facilius exui corporalibus possimus ex vinculis et tenebrosam evadere caecitatem. Verba sunt codicis, interpunctio editoribus debetur, qui cum plurimi suis ipsi inventis (interpunctionem dico) adquiescere non possent, in devia omnia abierunt. Integritatem tamen loci restituisse sibi visus est Hugius p. 23 et 24, qui acerrumus glossematum venator eiectis verbis adscribere infortunio voluptatem, modo cum Heraldo eam ante voces nec timere nec fugere intercidisse statuas, sarta tecta esse adfirmat. At vir doctissumus de usu Arnobiano, nedum de latino 
securior fuisse videtur. Quid enim aestimare fortnnae? oportebat, nisi fallor, existimare. Atqui ne boc quidem Arnobianum est, qui habendi modo et videndi verba cum eiusmodi genetivo iungere soleat. Itaque cum hac quidem via incedere non liceat, alia loco medicina ratione invenienda erit. Qua re ut ita interpungas auctor sum: quinimmo edocti sumus minas omnes quaecunque sunt parvi ducere atque aestimare, fortunae ac, si quando ingruerit vis quaepiam gravior, quam funem necesse sit consequi, adscribere infortunio voluptatem, nec timere nec fugere e. q. s. Verba timere et fugere alssoluta nihil habent quo offendaris. De persecutione enim agitur quam nec timendam nec fugiendam cum literis sacris patres vetustissumi docent.

II, 77 in.: ut si aliquis brutus ac stolidus in carcerem hominem $n a t u m$ in egressum quaestionum munquam adficere segregavimus atque immanibus existimet poenis, nisi in ipsum saeviat carcerem, materiam eius comminuat atque urat tectum, parietem, ianuas partesque alias operis renudet, deiciat, adfligat, nesciens hoc facto ei cui videatur officere dari ab se lucem et sceleratam eripi caecitatem. Haec codicis verba vix dici potest quantas interpretibus moverint turbas. Atque sine dubio nec natum inegressum nec segregavimus babent quo se tueantur. Bene Ursinus datum; quod autem inegressum, quasi glossa esset, eiecit, non bene fecit, etsi multo circunspectius quam 0eblerus, qui inegressibilem miro sane consilio interpretatur. Sequentia enim carcerem poni caecum, obscurum, cui deiecto demun tecto, eversis parietibus lumen reddatur, docent. Atque cum eiusmodi aliquid illi voci subesse appareat, scribendum censeo: in carcerem hominem datum. [lum]ine cassum. Quod posui cassum plane Arnobianum est, himine autem cassum Lucretianum (cf. IV, 369, V, 719). Praeterea quaestionum immanes pocnae quae sunt, quae esse possunt? Nibilominus editores omnes tertium illud vitium sublatum putaverunt, modo scriberetur se vel sese gravibus, praeter unum Hildebrandum, qui prudenter manum de tabula abstulit. Sanum praestiteris locum scribeudo: se gravaminibus.

II, 78 extr.: commitlamus nos deo nec plus apud nos valeat incredulitas nostra quam illius nominis et potentiae magnitudo, ne, dum ipsi nobis argumenta contuirimus, quibus "esse videatur falsum id quod esse nolimus atque abnutemus verum, obrepat dics extremus et inimicae mortis reperiamur in faucibus. Ita cum codice editio 
Lugdun., Hild., Oehlerus. Reliqui editores cum adnutemus verum latinum non esse optume perspexissent, adnitimur verum maluerunt. Ne hoc quidem recte. Locus plane contrario, quam quo II. 59 in. restituendum putavi, modo sanandus est: ibi enim cum rerum pro verum in codice exaratum inveniatur, boc loco verum male pro rerum scriptum est. Itaque locum aliter dispunctum sic enreudo: ne, dum ipsi nobis argumenta confuirimus, quibus esse videatur falsum id quod esse nolimus atque abnutemus, rerum obrepat dies extremus et inimicae mortis reperiamur in faucibus.

III, 1 med.: neque enim res stare sine adsertoribus non potest religio Christiana aut eo esse comprobabitur vera, si adstipulatores habuerit phurimos et auctoritatem ab hominibus sumpserit. Sic cum codice Oeblerus solus; Hildebrandus restare; reliqui neque enim stare s, a. n. potest et religio. Ipsum autem illud sine adsertoribus poscit ut emendetur: neque enim per se stare sine adsertoribus non potest religio christiana.

III, 3. in.: atque ut in terrestribus regnis necessitate mulla compellimur regalibus in familiis constitutos nominatim cum principibus adorare, sed in regum ipsorum cultu quidquid illis adnexum est tacita ut se sentit honorificentia comprachendi, non alia ratione e. s. q. Praeter Hildebrandum et Oelılerum omnes editores in altero ut offenderunt idque aut eiciendum aut at aut vi substituendum censuerunt. Sed nominatim adorare et tacitam honorificentiam cum quadam empliasi sibi opponi non animadverterunt. Corrige: tacita vel se sentit honorificentia comprachendi. De postposita particula ne dubites cavebit Hildebrandus ad II, 60,

III, 3. med.: si modo liquet et constat praeter ipsum regem et principem esse a lienum capita, quae digesta et separata per numerum velut quemdam populum plebeiae multitudinis faciant. Primus quid res posceret intellexit Rigaltius, esse alia numinum capita emendans, quem secuti Hildebrandus et 0eblerus esse alia deum capita maluerunt. Ad codicem proxume accedit: esse alienigenum capita, id quod, nisi fallor, etiam plebeia multitudo ut scribatur flagitat.

III, 3. extr.: neque nobis in aedibus sacris effigies pro diis illa et simulacra velitis ostendere qua intellegitis vos quopue et recusatis et rentitis confiteri vilissimi esse formas luti et fabrorum figmenta puerilia. Cum codice faciunt ed. pr. Urs. Orell. Hild. 
0ehl.; reliqui et illa traiciunt. Emendandum esse pro diis [sig]illa et simulacra tam est manifestum, ut verbis parcere satius ducam. In sequeutibus at recusatis cum edit. Lugdunensi maluit Oehlerus, sel vel nec alii. Equidem malin: etsi recusatis.

(Continuabuntur).

Dabam Rudolphopoli.

Ern. Klussmann.

\section{Zu Homer's Ilias.}

Die alten haben Hom. Il. $\Phi, 10 \mathrm{flg}$. immer beachtet, die neuern weniger: erst Hoffmann sucht die erklärung genauer zu begründen: . . oi $\delta^{\prime} \quad \vec{a} \lambda(\lambda \lambda \eta \eta \vec{\omega}$

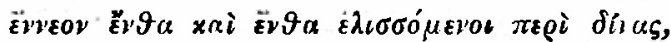

wie jetzt die ausgaben: Heyne und so früher überhaupt xatà $\delta$ $\nu a c$, meines erachtens richtig. Denn $\mathbf{X}$ anthos wird hier nachdrücklichst als reissender strom geschildert: er und seine ufer tönen, vs. 16. 11, er ist tief, voll von strudeln, vs. 2. 8, was $\beta \alpha \vartheta v \delta(\nu \eta \varsigma$ vs. 15 vereinigt: in ihn plumpen lediglich aus furcht die Trojaner und schwimmen है้ $9 a$ xai है $\vartheta \alpha$, d. h. ohne vorwärts zu kommen (vrgl. Nägelsb. Erkl. anm. zu Il. II, 397), was durch $\varepsilon \lambda \imath \sigma \sigma o ́ \mu \varepsilon r o b$, das passivische, erklärt wird: die gewalt des stromes zwingt zu dem vergeblichen streben: es gehört also $\ddot{\varepsilon} \nu \vartheta a$ xai $\ddot{\varepsilon} \nu \vartheta a$ zum verbum finitum wie zum particip, das will wohl Schol. ad vs. 11 sa-

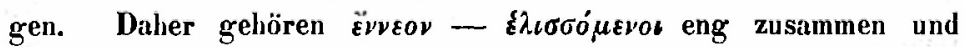
sind durch kein komma zu scheiden, wie Doederl. Hom. Gloss. II. p. 39 angedeutet, in der ausgabe aber aufgegeben hat: zu diesem

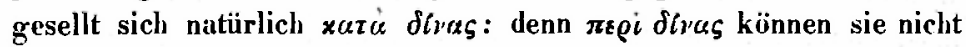
schwimmen, weil dann sie ihrem willen folgten; ausserdem ist der strom voll von wirbeln, so dass $\pi \varepsilon g i$ ganz gegen die natur der

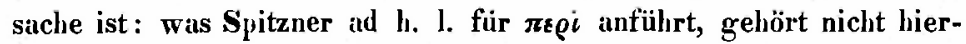
her: endlich wird xazù über allen zweifel erhoben durch inf. 353:

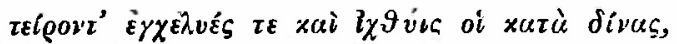

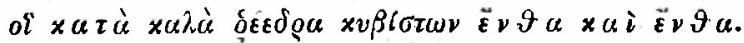

Das rs. 12 folgende gleichniss bezieht sich nicht auf diese stelle, sondern hat nur das vor vs. 10 stehende im auge.

Ernst von Leutsch.

Philologus. XXVI. Bd. 4. 